Bio - grafía. Escritos sobre la Biología y su Enseñanza. ISSN 2027-1034

Edición Extraordinaria. p.p. 525 - 532

Memorias del IX Encuentro Nacional de Experiencias en Enseñanza de la Biología y la Educación Ambiental. IV Congreso Nacional de Investigación en Enseñanza de la Biología.

\title{
LAS CONCEPCIONES SOBRE AMBIENTE Y PROBLEMÁTICA AMBIENT AL QUE POSEEN LOS MAESTROS Y ESTUDIANTES: UN DIAGNÓSTICO PARTICIPATIVO Y UNA PROPUESTA DIDÁCTICA
}

\author{
CONCEPTIONS ABOUT ENVIROMENT AND EMVIROMENTAL ISSUES THAT \\ TEACHERS AND STUDENTS HAVE: A PARTICIPATORY DIAGNOSIS AND A \\ DIDACTIC PROPOSAL
}

\author{
Valencia Katerin \\ Cardona Juan Diego ${ }^{1}$
}

\section{RESUMEN}

Este trabajo describe los resultados de un estudio exploratorio sobre las concepciones de ambiente y problemática ambiental de maestros y estudiantes de la Institución Educativa José Horacio Betancur, perteneciente al corregimiento de San Cristóbal Medellín. Los participantes manifiestan entre otras cosas que, la biodisponibilidad, el acceso y el tratamiento del agua para las actividades de la vida cotidiana son uno de los principales problemas que aquejan a la comunidad educativa, de ahí que, a la luz de estos hallazgos se construye una unidad didáctica dirigida a los estudiantes del grado quinto con el fin de abordar las temáticas relacionadas con el ciclo del agua y la separación de mezclas desde una perspectiva de la educación ambiental. El objetivo de esta unidad es comprender las alteraciones provocadas al ciclo del agua a causa de actividades socio-culturales y conocer las medidas que se pueden tomar para disminuir estos impactos. La propuesta didáctica toma como estrategia principal la resolución de problemas del contexto para que los estudiantes no sólo asuman posiciones críticas frente a la escases mundial del agua, sino que también posean herramientas para solucionar diversas situaciones problema de la comunidad.

PALABRAS CLAVE: enseñanza del agua, educación ambiental, problemas ambientales.

\section{ABSTRACT}

This paper describes the results of an exploratory study on environmental conceptions and environmental problems of teachers and students of the Educational Institution José Horacio Betancur, belonging to the district of San Cristóbal-Medellín. The participants state among other things that the bio availability, access and treatment of water for activities of daily living are one of the main problems that afflict the educational community, hence, in light of these findings are Constructs a didactic unit directed to the students of the fifth grade in order to approach the themes related to the water cycle and the

\footnotetext{
${ }^{1}$ Universidad de Antioquia
} 
Bio - grafía. Escritos sobre la Biología y su Enseñanza. ISSN 2027-1034

Edición Extraordinaria. p.p. 525 - 532

Memorias del IX Encuentro Nacional de Experiencias en Enseñanza de la Biología y la

Educación Ambiental. IV Congreso Nacional de Investigación en Enseñanza de la Biología.

separation of mixtures from an environmental education perspective. The objective of this unit is to understand the alterations caused to the water cycle due to socio-cultural activities and to know the measures that can be taken to reduce these impacts. The main objective of the didactic proposal is to solve problems of the context so that students take not only critical positions in relation to the world water shortage, but also have tools to solve various problem situations of the community.

KEYWORDS: teaching about water, environmental education, environmental problems.

\section{INTRODUCCIÓN}

La educación ambiental como área transversal al currículo que se desarrolla en los establecimientos educativos a través de un proyecto pedagógico; precisa el abordaje de situaciones que ubiquen los tópicos y contenidos a partir de problemas del entorno y contexto escolar. Se asume una perspectiva del ambiente en la cual la realidad se aprecia como un entramado complejo de relaciones entre los sistemas físicos, naturales y socioculturales. Partiendo de la relación de las experiencias cotidianas que viven los estudiantes y el medio en el que ellos se desenvuelven con los saberes y competencias que se enseñan, otorgando de esta manera un nuevo sentido a la enseñanza-aprendizaje de la educación ambiental y las ciencias (López, 1999).

La importancia de la educación ambiental radica en el hecho de ser necesaria para tomar conciencia sobre los impactos de las interacciones que hay entre los diferentes componentes del ambiente. Donde el ser humano es uno de los grandes causantes de diversas alteraciones que pueden llevar a la destrucción del entorno, las formas de vida y las sociedades.

Ahora bien, la educación ambiental es imprescindible en una sociedad donde su sistema económico está basado en el uso irracional y sin límites de recursos naturales para la producción desmedida de bienes y servicios. Comprometiendo no sólo la supervivencia de las generaciones actuales sino también la de las futuras (Vega, Freitas, Álvarez, \& Fleuri, 2009).

Transversalizar los conocimientos y habilidades para la inclusión de la educación ambiental en el aula de ciencias favorece en los estudiantes la construcción de una noción de ambiente y sistema ambiental que integra al ser humano -y su entramado social- con las dinámicas del entorno natural (Diniz \& Tomazello, 2005). De esta forma entonces, la educación ambiental se convierte en un puente en el que confluyen saberes de la ciencia y de la vida cotidiana, para alcanzar el principio de integralidad que pretende la formación de los ciudadanos en la educación regular. Independiente de la estrategia que se utilice para la integración de la educación ambiental al currículo -como asignatura, proyecto, actividad puntual, campaña, elemento de otra asignatura, entre otras- Gayford (1998) manifiesta que éstas deben incorporar los factores bio-físico, económicos, 
Bio - grafía. Escritos sobre la Biología y su Enseñanza. ISSN 2027-1034

Edición Extraordinaria. p.p. 525 - 532

Memorias del IX Encuentro Nacional de Experiencias en Enseñanza de la Biología y la

Educación Ambiental. IV Congreso Nacional de Investigación en Enseñanza de la Biología.

sociales, estéticos, políticos, espirituales, científicos y tecnológicos ligados con la dimensión y las situaciones ambientales.

En el ámbito colombiano, apreciamos ya desde la Constitución Política Nacional del año 1991 algunos enunciados de derechos básicos en los cuales se hace evidente la responsabilidad de la escuela en la formación ambiental de los ciudadanos. En este sentido, se contempla en el artículo 67 que "la educación formará al colombiano en el respeto a los derechos humanos, a la paz y a la democracia; y en la práctica del trabajo y la recreación, para el mejoramiento cultural, científico, tecnológico y para la protección del ambiente". Aunque la educación juega un papel indispensable en la alfabetización ambiental de las personas, el Estado se impone como tarea adjunta "la planificación y aprovechamiento de los recursos naturales, para garantizar su desarrollo sostenible, su consenvación, restauración o sustitución" (Ibíd., Art. 80), pero también proscribe el deber que tienen los sujetos de "proteger los recursos culturales y naturales del país y velar por la conservación de un ambiente sano" (Ibíd., Art. 95, numeral 8).

Los planteamientos constitucionales se concretan en algunos de los propósitos de la educación básica y media estipulados en la Ley 115 de 1994 al establecer para la educación formal los siguientes objetivos generales:

- "Proporcionar una formación general mediante el acceso, de manera crítica y creativa, al conocimiento científico, tecnológico, artístico y humanístico y de sus relaciones con la vida social y con la naturaleza, de manera tal que prepare al educando para los niveles superiores del proceso educativo y para su vinculación con la sociedad y el trabajo" (República de Colombia, 1994, Art. 20, literal a)

- "La comprensión básica del medio físico, social y cultural en el nivel local, nacional y universal, de acuerdo con el desarrollo intelectual correspondiente a la edad" (lbíd., Art. 21 literal f).

- "La valoración de la higiene y la salud del propio cuerpo y la formación para la protección de la naturaleza y el ambiente" (Ibíd., Art. 21, literal h).

- "El desarrollo de actitudes favorables al conocimiento, valoración y conservación de la naturaleza y el ambiente" (Ibíd., Art. 22, literal e).

Cada uno de estos objetivos configuran el marco a partir del cual la escuela hará frente a la realidad ambiental, reflexionando críticamente respecto a las relaciones dialógicas Hombre- Naturaleza- Ciencia- Tecnología - Sociedad - Ambiente a través de una concepción filosófica cimentada en los principios fundamentales de la educación ambiental formulados en la conferencia de Tbilisi: a) la concientización, b) la formación en valores, actitudes y comportamientos, c) la adquisición de conocimientos, d) la adquisición de competencias, y e) la participación.

Recogiendo las ideas anteriores, concebimos entonces la educación ambiental como "el proceso en el que los individuos y la colectividad cobran conciencia de su medio y adquieren los conocimientos, los valores, las competencias, la experiencia y la voluntad 
Bio - grafía. Escritos sobre la Biología y su Enseñanza. ISSN 2027-1034

Edición Extraordinaria. p.p. 525 - 532

Memorias del IX Encuentro Nacional de Experiencias en Enseñanza de la Biología y la Educación Ambiental. IV Congreso Nacional de Investigación en Enseñanza de la Biología.

capaces de hacerlos actuar individual y colectivamente para resolver los problemas actuales y futuros del ambiente" (Meinardi, Aduríz-bravo \& Revel, 2002., p. 94).

\section{METODOLOGÍA}

Desde el punto de vista metodológico este trabajo se sustenta en el paradigma socio crítico, porque trasciende el solo hecho de recolectar información sobre las concepciones de los integrantes de la comunidad educativa, proyectándose también como una estrategia para transformar las ideas de los estudiantes y desarrollar habilidades, conocimientos y actitudes en el campo de las ciencias y la educación ambiental a través de una unidad didáctica sobre el ciclo del agua como propuesta educativa. En la etapa en la que nos encontramos tiene el alcance de un estudio exploratorio transversal, porque la información se obtiene en un fragmento temporal definido -primer semestre- y porque los datos se analizan descriptivamente y se obtienen a partir de la aplicación de un cuestionario con preguntas abiertas a 16 estudiantes ( 8 de primaria y 8 de bachillerato) y 18 profesores de primaria y bachillerato. Las preguntas del cuestionario indagan sobre la concepción de ambiente y problemática ambiental que poseen estos integrantes de la comunidad educativa. Cabe resaltar que las preguntas del cuestionario de los estudiantes se adaptan de acuerdo a su grado y edad con el fin de facilitar la comprensión de quienes lo diligencian. El trabajo de campo se lleva a cabo en la Institución Educativa José Horacio Betancur; ubicada en el corregimiento de San Cristóbal (Medellín), en la vereda La loma. Esta comunidad es de estrato socio económico bajo y las construcciones están asentadas en terrenos inestables y riesgosos (constantes deslizamientos).

\section{RESULTADOS}

Tabla 1 Codificación subcategorías concepción de problemática ambiental

Subcategorías de la concepción de problemática ambiental

\begin{tabular}{lll}
\hline Codificación & Factor desencadenante & \multicolumn{1}{c}{ Dimensión afectada } \\
\hline DesBio-DimBio & Entorno biofísico & Entorno biofísico \\
DesBio-DimH & Entorno biofísico & Entorno humano \\
DesH-DimBio & Acciones humanas & Entorno biofísico \\
Desh-DimH & Acciones humanas & Entorno humano \\
DesInt-DimHBio & Interacción de las acciones & Entorno humano y biofísico \\
& humanas y elementos del & \\
& entorno biofísico & \\
\hline
\end{tabular}


Bio - grafía. Escritos sobre la Biología y su Enseñanza. ISSN 2027-1034

Edición Extraordinaria. p.p. 525 - 532

Memorias del IX Encuentro Nacional de Experiencias en Enseñanza de la Biología y la

Educación Ambiental. IV Congreso Nacional de Investigación en Enseñanza de la Biología.

\section{Concepción de ambiente}

Con respecto a la concepción de ambiente que poseen los maestros y estudiantes, los resultados muestran que la mayoría de estudiantes piensan que el ambiente se reduce al espacio biofísico que les rodea, fue común entre sus respuestas encontrar expresiones como "Es todo aquello que nos rodea específicamente la naturaleza". Por otra parte $7 \mathrm{de}$ los 16 estudiantes presentan dificultades para definir el concepto de ambiente tal y como lo evidencia la tabla 2. Ahora con respecto a la concepción de ambiente de los profesores, se puede evidenciar que 11 de los 18 maestros poseen una perspectiva biofísica del ambiente, mientras que 7 de los maestros poseen una concepción holística. Algunos de estos últimos docentes creen que el ambiente "son todas las interacciones sociales, económicas, políticas y naturales que nos afectan".

Tabla 2 Frecuencia de las concepciones de los participantes según las categorías

\begin{tabular}{|c|c|c|c|c|c|c|c|c|}
\hline \multirow{3}{*}{$\begin{array}{c}\text { Participant } \\
\text { e }\end{array}$} & \multicolumn{8}{|c|}{ Concepciones } \\
\hline & \multicolumn{3}{|c|}{ ambiente } & \multicolumn{5}{|c|}{ Problema ambiental } \\
\hline & $\begin{array}{l}\text { Biofisic } \\
\text { a }\end{array}$ & $\begin{array}{l}\text { Holistic } \\
\text { a }\end{array}$ & $\begin{array}{l}\text { No } \\
\text { respond } \\
\text { e }\end{array}$ & $\begin{array}{l}\text { DesH- } \\
\text { DimBI } \\
0\end{array}$ & $\begin{array}{l}\text { DesBio } \\
\text {-DimH }\end{array}$ & $\begin{array}{l}\text { Deslnt- } \\
\text { DimHBi } \\
0\end{array}$ & $\begin{array}{l}\text { Desh } \\
- \\
\text { Dim } \\
\text { H }\end{array}$ & $\begin{array}{l}\text { No } \\
\text { respond } \\
\text { e }\end{array}$ \\
\hline Estudiante & 11 & - & 7 & 12 & - & - & - & 6 \\
\hline Profesor & 11 & 5 & - & 14 & 2 & 2 & - & - \\
\hline
\end{tabular}

\section{Concepción de problemática ambiental}

En cuanto a la concepción de problemática ambiental las respuestas se clasificaron en 4 subcategorías planteadas por Cardona (2012) en su tesis doctoral. Estas subcategorías son codificadas como se muestra en la tabla 1. Ahora, con respecto a los resultados se evidencia que la mayoría de estudiantes (12 en total) considera que las problemáticas ambientales son desencadenadas por las acciones humanas las que afectan directamente al entorno biofísico. Asimismo, en el caso de los maestros, 14 de estos presentan la misma concepción de problemática ambiental descrita. Sin embargo, 2 de los docentes consideran que las problemáticas ambientales son producidas por las acciones humanas y afectan el entorno humano. Cabe resaltar que solo 2 docentes de los 18 participantes consideran que las problemáticas ambientales son desencadenadas por la interacción de las acciones humanas y los elementos del entorno biofísico lo cual afecta tanto lo humano como el entorno.

Por otra parte, ninguno de los participantes consideran que las problemáticas ambientales se generan por el entorno biofísico y afectan solo este espacio. Igualmente, ningún 
Bio - grafía. Escritos sobre la Biología y su Enseñanza. ISSN 2027-1034

Edición Extraordinaria. p.p. 525 - 532

Memorias del IX Encuentro Nacional de Experiencias en Enseñanza de la Biología y la

Educación Ambiental. IV Congreso Nacional de Investigación en Enseñanza de la Biología.

maestro ni estudiante considera que el origen de la problemática ambiental se da en el entorno biofísico afectando al ser humano.

\section{Problemáticas ambientales institucionales}

En la tabla 3 encontramos un resumen de las problemáticas ambientales de la institución reconocidas por los participantes luego del análisis de su contexto mediante un taller de grupo focal.

Tabla 3 Problemáticas ambientales institucionales y estrategias de solución

Problemática reconocida Estrategia de solución

Manejo de residuos sólidos $\quad$ Reciclaje, organización y limpieza

Uso del agua

Campañas y carteles alusivos a la conservación del agua

Disposición zonas verdes

Cuidado y mantenimiento de los jardines

En cuanto a las problemáticas ambientales institucionales reconocidas por los participantes; estas visiones se orientan por las ideas frecuentemente trabajadas en la educación ambiental (cuidado del agua y residuos sólidos). Concepciones muy relacionadas con la percepción naturalista del ambiente; haciéndose evidente una real problemática de fondo y es la falta de comprensión del concepto de ambiente y las interacciones socio-culturales con el entorno y sus tensiones.

\section{CONCLUSIONES}

Dado que la propuesta de enseñanza se implementará durante el segundo semestre del presente año, aún no es posible presentar resultados sobre la incidencia que ha tenido en la evolución de las concepciones de los estudiantes y el desarrollo de actitudes proambientales. Por lo anterior, las conclusiones versan sobre el estudio exploratorio.

Haciendo referencia a las concepciones del ambiente que poseen los estudiantes se puede concluir que estos poseen visiones que reducen el ambiente a los espacios biofísicos sin concebir las interacciones que se llevan a cabo entre los factores y componentes del ambiente. Asimismo, fue evidente que algunos de los estudiantes presentan dificultades para definir el concepto de ambiente, por lo que se concluye que es necesario implementar estrategias de educación ambiental que apunten a fortalecer este concepto tanto en maestros como en estudiantes. 
Bio - grafía. Escritos sobre la Biología y su Enseñanza. ISSN 2027-1034

Edición Extraordinaria. p.p. 525 - 532

Memorias del IX Encuentro Nacional de Experiencias en Enseñanza de la Biología y la Educación Ambiental. IV Congreso Nacional de Investigación en Enseñanza de la Biología.

Con respecto a los maestros, la mayoría tiene una perspectiva biofísica del concepto de ambiente. Sin embargo algunos de los docentes presentan la visión holística del concepto independientemente de su formación profesional.

La mayoría de maestros y estudiantes consideran que las problemáticas ambientales son ocasionadas por las acciones humanas y afectan el entorno biofísico o al entorno humano. Lo que lleva a entender que no se da importancia a las interacciones y relaciones que se dan en el ambiente y que afectan a todos sus componentes directa 0 indirectamente.

Es por tal razón, que se diseña una unidad didáctica cuya temática aborda el ciclo del agua en el marco de la crisis mundial por la escases del recurso. Esta temática se seleccionó debido a que fue una de las problemáticas institucionales reconocidas por los participantes. Sin embargo, este tema es un pretexto para socavar la dificultad relacionada con la falta de comprensión del concepto de ambiente y las relaciones socioculturales con el entorno. La unidad didáctica consta de 8 sesiones donde se implementa la estrategia de la resolución de problemas bajo el enfoque de educación ambiental para la sostenibilidad. El público objetivo son los estudiantes del grado quinto, y su desarrollo articula el PRAE y los contenidos propios del área de ciencias naturales.

Para el abordaje de los saberes y competencias se parte de dos preguntas problema que los estudiantes resolverán durante el proceso de implementación : ¿Qué relaciones entre el hombre y la naturaleza generan problemáticas relacionadas con el uso del agua y su ciclo? Y ¿De qué forma el conocimiento sobre la separación de las mezclas puede ayudar a solucionar la problemática de la contaminación del agua? Estas preguntas se dinamizan a través de las siguientes actividades por fases del ciclo didáctico:

- Exploración de ideas previas: a) Actividad 1: Los dinosaurios tomaron la misma agua que consumimos hoy en día, b) Actividad 2 ¿y cómo obtengo la sal?

- Introducción de nuevos conocimientos: c) Actividad 3: Analizando los datos críticos sobre el uso del agua a nivel mundial, d) Actividad 4 Construcción de un mapa grafico de ideas, e) Actividad 5 Reevaluando las ideas, f) Actividad 6 ¿Cómo se altera el ciclo del agua?

- Estructuración y síntesis:, g) Actividad 7 Reconozco los problemas de mi comunidad, h) Actividad 8 ¿Y qué hacer con el problema mundial del agua?, i) Actividad 9 Exploración de animación sobre separación de mezclas.

- Aplicación: Actividad 10 Proponiendo soluciones a problemas del contexto. 
Bio - grafía. Escritos sobre la Biología y su Enseñanza. ISSN 2027-1034

Edición Extraordinaria. p.p. 525 - 532

Memorias del IX Encuentro Nacional de Experiencias en Enseñanza de la Biología y la Educación Ambiental. IV Congreso Nacional de Investigación en Enseñanza de la Biología.

\section{BIBLIOGRAFÍA}

-Cardona, J.D. (2012). Concepciones sobre educación ambiental y desarrollo profesional del profesorado de ciencias experimentales en formación. Huelva: Universidad de Huelva.

-Delatorre, A., Del Rincón, D. y Arnal, J. (1997). Bases metodológicas de la investigación educativa. Primera reimpresión. Barcelona: Hurtado ediciones.

-, E.M. y Tomazello, M.G. (2005). A pedagogia da complexidade e o ensino de conteúdos atitudinais na educação ambiental. Revista Eletrônica do Mestrado em Educação Ambiental, 15, 80-93.

Enlace web: http://www.remea.furg.br/edicoes/vol15/art07.pdf.

-López, R. (1999). La investigación de problemas ambientales orientada a la resolución de los mismos, como un posible modelo de incorporación de la educación ambiental en el curriculum. Innovación Educativa, 9, 269-280.

-Martínez, M. (1998). La investigación cualitativa etnográfica en educación: manual teórico-práctico. Tercera edición. México: Trillas.

-Meinardi, E., Adúriz-bravo, A. y Revel, A. (2002). La educación ambiental en el aula. Una propuesta para integrar contenidos multidisciplinares a través de la argumentación. Investigación en la Escuela, 46, 93-103.

-República de Colombia (1991). Constitución Política Nacional. Bogotá: Senado de la República.

-República de Colombia (1994). Ley General de Educación o Ley 115. Bogotá: Ministerio de Educación Nacional.

-Vega, P., Freitas, M., Álvarez, P., \& Fleuri, R. (2009). Educación ambiental e intercultural para la sostenibilidad: fundamentos y praxis. Revista internacional de filosofía iberoamericana y teoría social, 14(44), 25-38. 